EXTENDED REPORT

\title{
Liposomal targeting of glucocorticoids to synovial lining cells strongly increases therapeutic benefit in collagen type II arthritis
}

\author{
J M Metselaar, W B van den Berg, A E M Holthuysen, M H M Wauben, G Storm, P L E M van Lent
}

Ann Rheum Dis 2004;63:348-353. doi: 10.1136/ard.2003.009944

See end of article for authors' affiliations

Correspondence to:

Dr P van Lent, Department

of Rheumatology,

University Hospital

Niimegen, University

Medical Centre 189, Geert

Grooteplein 26-28, 6500

HB Nijmegen, The

Netherlands;

P.vanLent@reuma.umcn.nl

Accepted

9 September 2003
Objective: To investigate the effect of a single intravenous treatment with glucocorticoids (GC) encapsulated in long-circulating PEG-liposomes on both joint inflammation and cartilage destruction and to investigate the phenomenon of selective homing of these liposomes in the inflamed synovium.

Methods: Mice with collagen type II-induced arthritis (CIA) were intravenously treated with liposomal and free prednisolone phosphate (PLP) a few days after the first signs of the disease. Joint inflammation was scored during 1 week after treatment, after which sections of the knee joints were prepared for assessment of cartilage damage. In addition, arthritic mice were treated with liposomes containing colloidal gold. 24 hours after injection, knee joint sections were prepared in which the location of liposomes was visualised.

Results: Treatment of CIA with $10 \mathrm{mg} / \mathrm{kg}$ liposomal PLP resulted in a strong and lasting resolution of joint inflammation. $10 \mathrm{mg} / \mathrm{kg}$ free PLP only became slightly effective after repeated daily injections. Although joint inflammation recurred 1 week after treatment with liposomal PLP, knee joint sections prepared at this time indicated that the cartilage damage was still reduced. Localisation of gold labelled liposomes in the inflamed joints was seen in the proximity of blood vessels, in the cellular infiltrate, but mainly in the synovial lining. Unaffected joints did not take up liposomes.

Conclusions: By using the property of long-circulating liposomes to target the synovial lining selectively in inflamed joints, the anti-inflammatory activity of GC can be greatly increased, showing also the beneficial effect of reduced cartilage destruction.
R heumatoid arthritis (RA) is a chronic autoimmune disorder, characterised by joint inflammation and cartilage destruction. ${ }^{12}$ An important role in the pathogenesis of joint inflammation is ascribed to the synovial lining layer that surrounds the connective tissue in the joints. Normally, the synovial lining consists of a few cell layers of mainly fibroblast-like and macrophage-like synoviocytes. In RA, however, the synovial lining layer expands as a result of newly arrived macrophages from the periphery. ${ }^{34}$ Macrophages in the synovial lining of arthritic joints have been shown to produce many proinflammatory cytokines, attract new inflammatory cells, and produce enzymes that can damage the cartilage. ${ }^{356}$

Liposomes were shown to be valuable for targeting macrophages as these cells efficiently phagocytose them upon exposure. ${ }^{7-9}$ Intra-articular administration of a liposomal form of pro-apoptotic clodronate selectively depleted the phagocytic lining cells, which resulted in a marked decrease of joint inflammation. ${ }^{10}{ }^{11}$ To realise macrophage depletion at inflamed synovia through the systemic route, Camilleri et al and Richards et al successfully employed small sized liposomes that localised in inflamed joints after intravenous (IV) injection. ${ }^{12-14}$ However, as most IV injected liposomes are generally taken up by the mononuclear phagocyte system (MPS) in liver and spleen, systemic treatment with liposomal clodronate may result in unintended elimination of hepatosplenic phagocytes, which play an important part in the immune defence of the body. ${ }^{14}{ }^{15}$

Effective suppression without elimination of synovial macrophages can be accomplished with glucocorticoids (GC). ${ }^{16}$ GC can strongly reduce the generation and release of proinflammatory cytokines and cartilage degrading enzymes by macrophages in arthritic joints. ${ }^{17}$ In fact, changes in endogenous steroid levels appear to play a part in the pathophysiology of RA. ${ }^{19}$ Besides suppression of activated macrophages, the proinflammatory activity of fibroblasts, lymphocytes, and endothelial cells is also suppressed, and this may explain the striking anti-arthritic activity of GC. However, serious adverse effects limit the systemic use of GC in arthritic patients. ${ }^{20} 21$ Moreover, high and frequent dosing (pulse therapy) is necessary to achieve sufficient activity in the joints, because target localisation is usually poor as a result of efficient clearance. ${ }^{22}$

For efficient delivery of GC into inflamed joints by systemic treatment, the use of small sized liposomes coated with poly(ethylene glycol) (PEG) may be advantageous. PEG has been shown to be very effective in reducing recognition and rapid removal of liposomes from the circulation by the MPS, enabling liposomes to stay in the circulation for a prolonged period of time. ${ }^{23-25}$ The long-circulation property provides the liposomes with the opportunity to substantially extravasate and accumulate in inflamed tissue. ${ }^{26}$

In a previous study we investigated the ability of PEGliposomes to selectively target GC to inflamed joints in rat experimental arthritis. The results of this study suggest that this may become a very valuable treatment approach. Joint inflammation could be completely resolved for a week after a single injection of liposomal prednisolone phosphate (PLP). In contrast, the same dose unencapsulated PLP was hardly

Abbreviations: CIA, collagen type II-induced arthritis; GC, glucocorticoid(s); IV, intravenous; MPS, mononuclear phagocyte system; PBS, phosphate buffered saline; PEG, poly(ethylene glycol); PLP, prednisolone phosphate; RA, rheumatoid arthritis 
effective, even when given daily. Additional whole body distribution studies showed that the increase of therapeutic efficacy is indeed a result of selective homing in the inflamed joints. ${ }^{27}$

The objective of our current study was to evaluate the capacity of long-circulating PEG-liposomes to target the inflamed synovium and to expose phagocytic cells in the lining to encapsulated GC. In the murine model of type II collagen-induced arthritis (CIA) the effect of liposomal GC on paw inflammation was investigated and compared with the effect of unencapsulated ("free") GC. Knee sections were prepared to evaluate the effect of liposomal GC on cartilage damage. Gold labelled liposomes were used to determine the exact location of uptake in the inflamed tissue and to evaluate whether liposome localisation is indeed inflammation driven.

Our results indicate that selective targeting of the inflamed synovial lining can be realised with long-circulating PEGliposomes. Encapsulation of GC in these liposomes dramatically increases the anti-inflammatory activity of GC in the inflamed synovium. Besides a complete resolution of paw inflammation, a significant reduction of cartilage damage, still visible 1 week after treatment, was achieved. The approach of targeted delivery to the inflamed synovium mediated by PEG-liposomes may strongly improve the value of GC in the treatment of arthritis.

\section{METHODS}

\section{PEG-liposomes containing GC}

Liposomes were prepared by the film extrusion method. ${ }^{28}$ Briefly, a lipid solution was prepared in ethanol, containing dipalmitoyl phosphatidylcholine (Lipoid GmbH, Ludwigshafen, Germany), PEG 2000-distearoyl phosphatidylethanolamine, and cholesterol (Sigma Chemical Co, Poole, UK) in a molar ratio of 1.85:0.15:1.0. The lipid solution was transferred to a round bottomed flask, and a lipid film was created by rotary evaporation. The film was hydrated with a solution of $100 \mathrm{mg} / \mathrm{ml}$ PLP in sterile water. The resulting lipid dispersion was sized by multiple extrusion through polycarbonate filter membranes. Unencapsulated prednisolone was removed by dialysis against $0.9 \%$ phosphate buffered saline (PBS) using Slide-A-Lyzer dialysis cassettes with a molecular weight cut off point of 10000 (Pierce, Rockford, IL, USA). Mean particle size was determined by dynamic light scattering with a Malvern 4700 system (Malvern Ltd, Malvern, UK). The liposomes were sized to a diameter between 90 and $100 \mathrm{~nm}$. Phospholipid content was determined with a phosphate assay ${ }^{29}$ in the organic phase after extraction of liposomal preparations with chloroform. The aqueous phase after extraction was used to determine the PLP content. With high performance liquid chromatography using a mobile phase of acetonitrile-water with $\mathrm{pH} 2$, connected to a UV detector, which was set at $254 \mathrm{~nm}$, both prednisolone and its phosphate ester could be measured in one single run. Each millilitre of the liposomal preparation contained around $4.5 \mathrm{mg}$ PLP and an average of $60 \mu \mathrm{mol}$ phospholipid.

\section{Gold labelled PEG-liposomes}

PEG-liposomes containing colloidal gold were prepared as described by Huang et al with some modifications. ${ }^{30}$ The method of preparation is similar to the method described above for the preparation of PLP-PEG-liposomes except for the hydration step, which was performed with a freshly prepared tetrachloroaurate solution in citrate buffer. Sizing was performed at $4{ }^{\circ} \mathrm{C}$ as described above. Immediately after extrusion, colloidal gold was formed by incubation of the dispersion at $37^{\circ} \mathrm{C}$. To remove non-encapsulated gold, the preparation was eluted on a Sephacryl S1000-SF column (Pharmacia, Uppsala, Sweden).

\section{Collagen-induced arthritis}

The Dutch Committee of Animal Experiments approved all animal studies. Male DBA/llacJ mice between 10 and 12 weeks of age (20-25 g) were obtained from Jackson Laboratories (Bar Harbor, ME, USA). To induce arthritis, $100 \mathrm{mg}$ of bovine type II collagen dispersed in complete Freund's adjuvant containing $2 \mathrm{mg} / \mathrm{ml}$ of heat inactivated Mycobacterium tuberculosis (both purchased from DIFCO laboratories, Detroit, MI, USA) was injected subcutaneously at the base of the tail. At day 21 a booster injection of $100 \mu \mathrm{g}$ of bovine type II collagen was given intraperitoneally. At day 24 after induction, the first signs of joint inflammation became visible. From that day on, the mice were regularly examined for visual signs of inflammation. The severity of the joint inflammation was "blindly" graded by assigning a score to each paw from 0 to 2 , based on erythema, swelling, and deformation of the joints. The sum of the grades for each animal is the clinical score and varies from 0 to $8 .{ }^{31}$

\section{Therapeutic efficacy}

All mice were treated on day 28 after induction, when the average score of all mice in the experiment is about half the maximal scores. On the day of treatment, groups of six to seven mice were formed with equal average clinical scores. $10 \mathrm{mg} / \mathrm{kg}$ PLP, both encapsulated in PEG-liposomes and in the unencapsulated form, was given as a standard dose. This dose was based on clinical experience with so-called "pulse" treatment of arthritic patients involving single or repeated injections of $1 \mathrm{~g}$ methylprednisolone. ${ }^{32}$ All preparations were given intravenously in the tail vein. For multiple injections of free GC, each following day the treatment was repeated at the same time for 5 days. The effect of treatment on clinical scores and body weight was monitored l week after treatment.

\section{Histology}

At 1 week after treatment knee joints were dissected and fixed in $4 \%$ formaldehyde in PBS. In addition, the joints were decalcified with 5\% formic acid in PBS during 7 days. After dehydration and embedment in paraffin, sections of the knee joint were cut that included the patella, femur, menisci, and tibia. These sections were mounted on gelatin coated microscopic slides, stained with haematoxylin and eosin, and examined using a light microscope (Leica, DMR, Germany). The arthritic score was determined by grading the infiltrate and the exudate from 0 to 3 , where $0=$ no cells; $1=$ minor cells; $2=$ moderate; $3=$ maximal number of cells within this experiment. Infiltrate is defined as the influx of leucocytes into the synovium. Exudate is defined as the influx of leucocytes into the joint cavity. Erosion of the cartilage surfaces was determined also using an arbitrary scale from 0 to 3 , where $0=$ no cartilage surfaces of the knee received a score (lateral and medial femur and lateral and medial tibia); $1=$ minor erosion; 2 = moderate erosion; 3 = maximal erosion. The scores of the erosion of the four surfaces were added. Scoring was performed in a "blinded" manner by two independent observers. The data represent the mean (SD) of eight animals.

\section{Visualisation of gold-liposomes}

A separate group of mice was treated with PEG-liposomes containing colloidal gold to visualise the exact location of the liposomes in the synovium. Twenty four hours after treatment knee joints were dissected and decalcified in EDTA/PVP (polyvinylpyrrolidine) in Tris buffer for 2 weeks. After freezing in liquid nitrogen, sections were cut in a cryostat 
(Microm, HM500M, Walldorf, Germany). These sections were mounted on Superfrost microscopic slides (Menzel Gläser, Germany). Silver enhancement of colloidal gold was performed with Sigma silver enhancer kit (Sigma, St Louis, MO, USA) and terminated by incubating with a $0.5 \%$ sodium thiosulphate solution in distilled water. The sections were then stained with haematoxylin and eosin and examined by light microscopy.

\section{Statistical analysis}

For statistically assessing and comparing therapeutic efficacy in different groups the non-parametric Wilcoxon/KruskalWallis test (rank sums) was used. For evaluating differences in infiltrate and exudate between groups, one way analysis of variance was used. For statistical evaluation of the effect on cartilage damage, Fisher's test was used. Values of $\mathrm{p}<0.05$ were considered significant.

\section{RESULTS}

\section{Therapeutic activity}

Figure IA shows the anti-inflammatory activity of free and liposomal PLP at a dose of $10 \mathrm{mg} / \mathrm{kg}$ in the murine CIA model. A single dose of free PLP did not have a significant effect on paw inflammation scores. However, the same dose encapsulated in PEG-liposomes resulted in a complete disappearance of paw inflammation at 5 days after treatment. In Figure 1B the effect of repeated daily injections of $10 \mathrm{mg} / \mathrm{kg}$ free PLP is shown in comparison with $10 \mathrm{mg} / \mathrm{kg}$ and $1 \mathrm{mg} / \mathrm{kg}$ liposomal PLP. Surprisingly, repeated administration with $10 \mathrm{mg} / \mathrm{kg}$ PLP also had only limited effect. The effect is significant compared with the control mice but not significantly different from the effect of a single injection of a 10-fold lower dose of liposomal PLP.

\section{Histological evaluation of inflammation and cartilage damage}

Figure 1 shows that 1 week after treatment paw inflammation scores were recurring. However, the knee sections prepared at this time still showed a profound therapeutic effect of liposomal PLP on the integrity of the cartilage layers (fig 2). In most of the tissue sections taken from control mice the cartilage appeared highly damaged, whereas in most of the treated mice loss of cartilage was hardly visible. Figure 3 shows only a small difference for the infiltrate and exudate as measures of inflammation. These results correspond with the recurrence of the inflammation score as shown in fig 1 . Scoring of the cartilage loss, however, disclosed a significant beneficial effect in the treated group (Fishers's test, $\mathrm{p}<0.05$ ). In four of the seven mice treated with liposomal PLP no cartilage degradation was detected, whereas all the control group mice showed profound loss of cartilage.

\section{Localisation of PEG-liposomes in the inflamed synovium}

Figures 4A and B show the localisation of PEG-liposomes containing colloidal gold in the knee sections of mice with CIA, visualised by silver enhancement. Microscopic evaluation showed that most of the liposomes accumulated in the region of the synovial lining. Most of the visualised gold seemed to be cell associated. In areas around certain blood vessels the density of liposomes appeared to be relatively high. Despite the severity of the arthritis model, knee joints in some mice were not inflamed. Interestingly, hardly any liposomes were visible in joints that did not show signs of inflammation (fig 4C). Clearly, the presence of an inflammatory process is a key factor in enabling liposomes to localise in the synovium.

\section{Localisation of PEG-liposomes in the spleen}

Silver enhancement of tissue sections from the spleen shows that almost all liposomes that were taken up by the spleen localised in the marginal zone and the red pulp. Visualised gold appeared to be largely cell associated. Hardly any gold was detected in the white pulp (fig 4D).

\section{DISCUSSION}

Previous results showed that encapsulation of PLP in longcirculating PEG-liposomes strongly increased the antiinflammatory activity of PLP as compared with the free drug. A single IV injection of $10 \mathrm{mg} / \mathrm{kg}$ liposomal PLP could induce complete resolution of rat adjuvant arthritis. Mechanistic studies with PLP encapsulated in different types
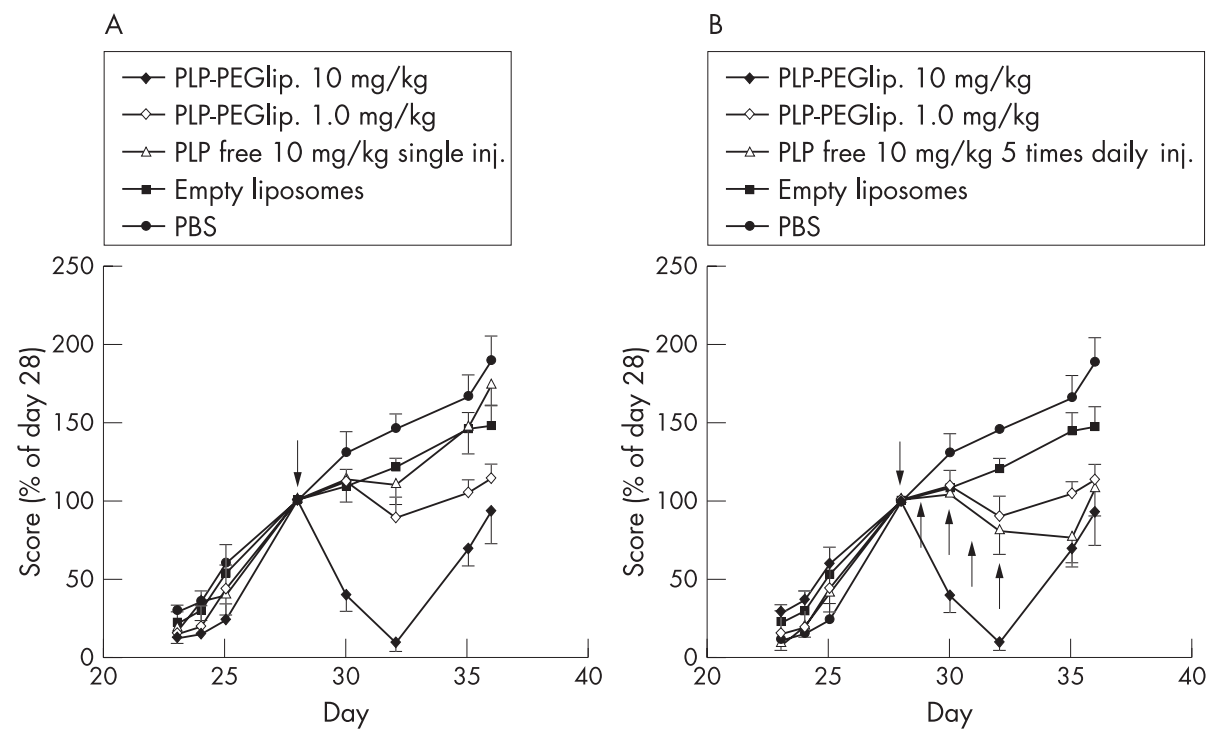

Figure 1 Paw inflammation scores after a single treatment with $10 \mathrm{mg} / \mathrm{kg}$ PLP-PEG-liposomes, $1 \mathrm{mg} / \mathrm{kg}$ PLP-PEG-liposomes, $10 \mathrm{mg} / \mathrm{kg}$ unencapsulated PLP, compared with both empty PEG-liposomes and PBS as controls. In contrast with liposomal PLP, a single treatment with $10 \mathrm{mg} / \mathrm{kg}$ unencapsulated PLP had no significant effect (A). Multiple treatment with five daily injections of $10 \mathrm{mg} / \mathrm{kg}$ unencapsulated PLP had a significant effect at days 32 and 35 but the effect was no better than that of $1 \mathrm{mg} / \mathrm{kg}$ PLP-PEG-liposomes (B). Each point represents the mean of 7 mice (SEM). Arrows indicate treatment. 

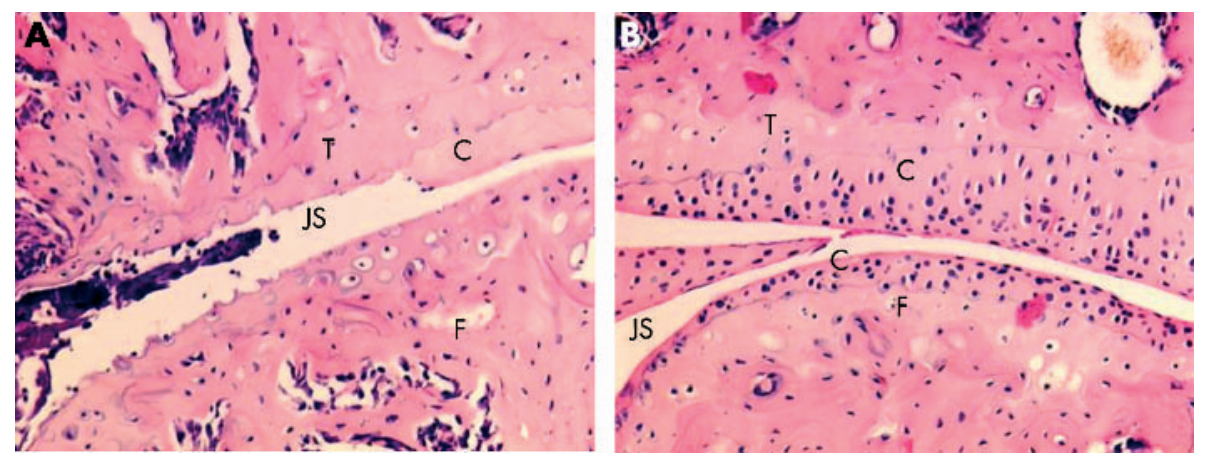

Figure 2 Effect of liposomal PLP on cartilage loss 1 week after treatment. (A) Knee joint section after treatment with saline; (B) the same knee joint after treatment with $10 \mathrm{mg} / \mathrm{kg}$ PLP-PEG-liposomes. Original magnification $\times 200$. Haematoxylin and eosin staining. T, tibia; $F$, femur, JS, joint space; $C$, cartilage layer.

of liposomes showed that the observed increased therapeutic efficacy was a result of the specific property of small longcirculating PEG-liposomes, which selectively homed to inflamed paws. Other explanations, such as uptake by MPS organs did not seem to contribute to the effect, and slow release of prednisolone from liposomes in the circulation did not occur at all. ${ }^{27}$ The present study focuses on the antiarthritic effect of PLP-PEG-liposomes in murine CIA, which is an experimental arthritis model with a different aetiology. In this model cartilage erosion is of major importance in the pathophysiology of arthritis, allowing a more in-depth investigation of the protective effect of liposomal PLP on cartilage by histological evaluation of inflamed joints. By histological examination the exact location of the PEGliposomes inside the inflamed synovium after extravasation from the vasculature could be visualised.

The data in this study confirm the profound antiinflammatory activity of PLP-PEG-liposomes. The reduction of paw inflammation scores after a single injection of $10 \mathrm{mg} /$ $\mathrm{kg}$ was rapid, and complete resolution of arthritis was achieved at day 5 after treatment. The therapeutic effect lasted for more than a week. $10 \mathrm{mg} / \mathrm{kg}$ unencapsulated PLP was only effective after repeated injection, but this strategy, which was based on the clinical "pulse" treatment regimen employed in patients with RA, was no more beneficial than a single injection of $1 \mathrm{mg} / \mathrm{kg}$ liposomal PLP. Although 1 week after treatment joint inflammation clearly recurred, cartilage erosion was still reduced in mice treated with PLP-PEGliposomes. Apparently, treatment with liposomal PLP profoundly delays the cartilage erosion process. This finding may be of importance as treatment of RA with IV "pulse" GC is

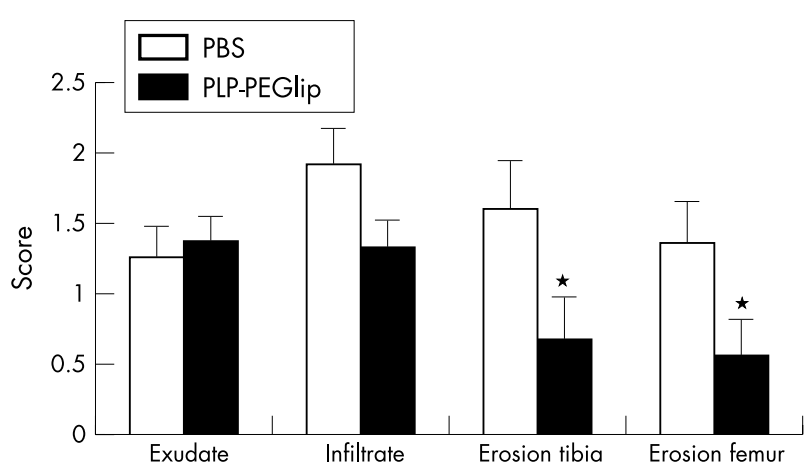

Figure 3 Histological evaluation of the effect on exudate and infiltrate as measures for inflammation, and on cartilage erosion, of $10 \mathrm{mg} / \mathrm{kg}$ liposomal PLP at 1 week after treatment. Data indicate the mean of seven mice (SEM). Asterisks indicate significance $(p<0.05)$. often criticised for its lack of ability to delay the progression of joint erosion. ${ }^{34} 35$

Knee joint histology performed after injection of gold labelled PEG-liposomes disclosed that the liposomes mainly localised in the synovial lining. Higher magnifications showed that almost all gold particles were cell associated. The fact that a clear distinction was seen between cells that are positive for gold staining and cells without intracellular gold, strongly suggests that PEG-liposomes selectively localised in cells with phagocytic capacity after extravasation in the inflamed synovium. As it was shown before that gold labelled PEG-liposomes are stable in vivo, it is assumed that all intracellular gold originated from phagocytosed liposomes. ${ }^{30}$ The affinity of liposomes for phagocytes was also clearly visible in the spleen, in which the phagocyte-rich red pulp showed strong liposome uptake, whereas the T cell-rich white pulp showed no sign of liposome uptake at all. As the PEG coating was used for its capacity to shield liposomes from recognition by MPS phagocytes, the extensive uptake by tissue phagocytes was somewhat unexpected. However, other researchers also report on similar observations with PEGliposomes targeted to sites of bacterial infection. ${ }^{36}{ }^{37}$

In addition to the synovial lining, some gold staining was also visible in the infiltrate and around the blood vessels, which suggests two possible explanations for the observed localisation of liposomes in the inflamed synovium. Firstly, the gold found in the inflamed synovium is associated with infiltrating monocytes/macrophages that phagocytosed liposomes in the periphery, or secondly, gold around some blood vessels is associated with liposomes extravasating from the circulation without the help of leucocytes. Schiffelers et al examined this issue and found support for the second explanation. ${ }^{36}$ Also, the fact that Camilleri et al and Richards et al report that their small liposomes containing clodronate eliminate synoviocytes despite the depletion of phagocytes elsewhere in the body, points in the direction of a localisation mechanism which is independent of phagocyte. ${ }^{12-14}$

As the onset of CIA varies, some knees of the CIA mice did not yet have signs of inflammation on the day of treatment. Tissue sections from these knee joints did not show any sign of liposome localisation. Clearly, PEG-liposomes show selectivity for inflamed synovia, which further supports the suggested role of inflammation enhanced vascular permeability as an essential phenomenon for liposome localisation.

The affinity of PEG-liposomes for macrophages in inflamed synovia makes them highly attractive carriers for GC. Macrophages have an important role in the onset and progression of arthritis, as they produce the proinflammatory cytokines tumour necrosis factor $\alpha$ and interleukins 1 and 6 , 

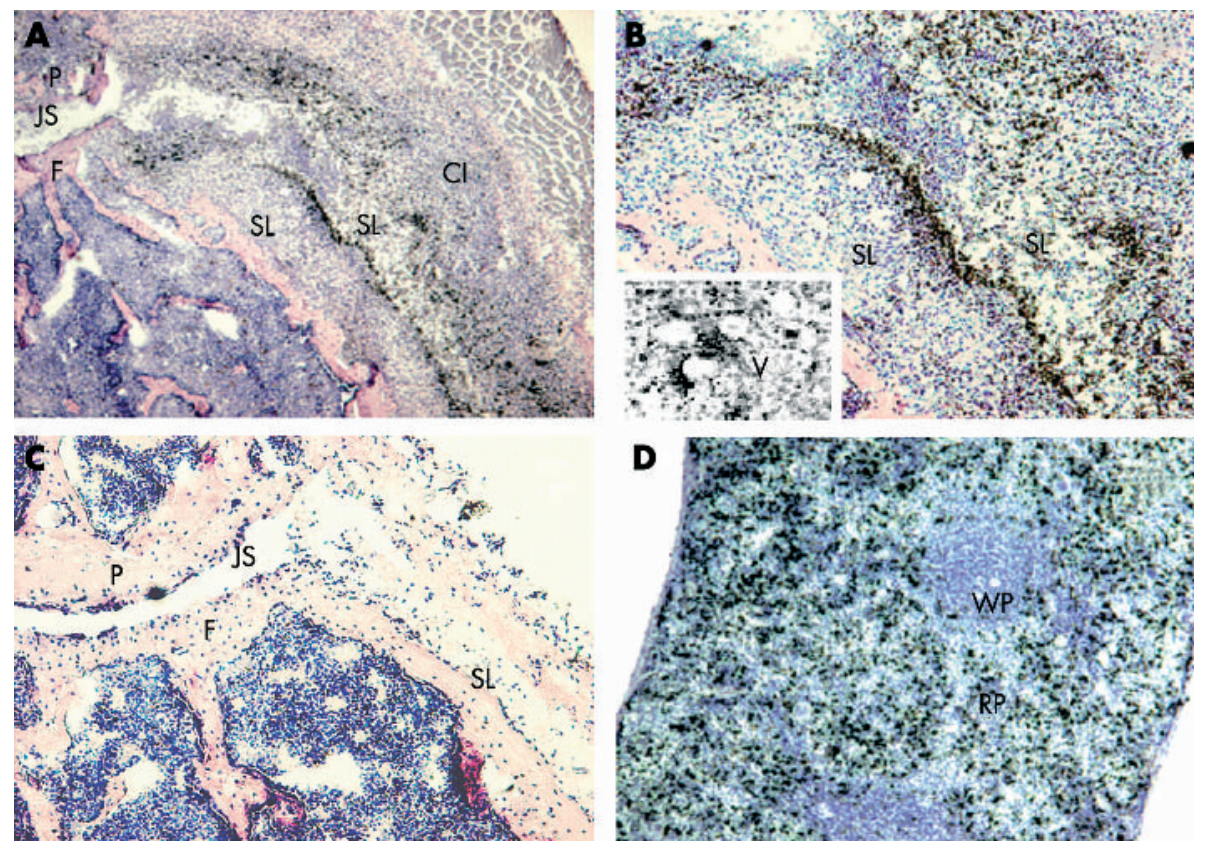

Figure 4 Visualisation of gold labelled PEG-liposomes. (A) Gold-liposomes in the inflamed knee joint. Original magnification $\times 50$. (B) Magnification $\times 100$ of an area surrounding the synovial lining. Insert: magnification $\times 200$ of an area surrounding blood vessels. Gold particles are visible as black dots. Note that the liposomal gold is mainly localised in the synovial lining and some around blood vessels. Relatively little gold is visible in the cellular infiltrate. (C) Visualisation of gold labelled PEG-liposomes in an unaffected knee joint. Original magnification $\times 50$. (D) Spleen localisation of gold labelled PEG liposomes. Original magnification $\times 100$. P, patella; F, femur, JS, joint space; Cl, cellular infiltrate; SL, synovial lining; V, vessels; WP, white pulp; RP, red pulp.

generate chemokines and tissue degrading enzymes, and have a role in the presentation of autoantigens to $\mathrm{T}$ cells. ${ }^{5}{ }^{6}$ Selective depletion of activated macrophages from the inflamed joint by intra-articular injection of liposomal clodronate has been shown to be a promising approach to treatment. ${ }^{10} 11$ Depletion of macrophages by the systemic route appeared to be less attractive, because elimination of macrophages from the liver and spleen was shown to strongly affect MPS functioning. ${ }^{15}$ GC may be more interesting to encapsulate in liposomes for a systemic treatment approach as GC can effectively down regulate the proinflammatory effector production without eliminating the macrophages.

The small PEG-liposomes used in this study offer the advantage of reduced uptake by the MPS, together with a prolonged circulation property, resulting in enhanced accumulation at inflamed sites. ${ }^{23-27}$ The extensive phagocytosis of PEG-liposomes we observed within the inflamed tissue will inevitably expose the macrophages to high intracellular concentrations of GC, which may be one plausible explanation for the strong anti-inflammatory effect. However, as GC can easily pass cellular membranes, the drug may escape from the intracellular compartment, suppressing other inflammatory cells in the synovium as well. Besides their role as target cells in arthritis, macrophages may therefore also have a crucial role in the release of GC from liposomes and the generation of relatively high and prolonged concentrations of active drug in the synovium. Previously, we showed that the liposomal formulation used in our studies was highly stable in vivo and that leakage of GC from the liposomes without the involvement of an external trigger is not likely. The suggested need for activated macrophages to release GC from liposomes may add an additional form of target selectivity to the liposomes besides selective accumulation at inflamed sites. Further studies are necessary to examine the uptake and intracellular processing of liposomal GC by macrophages.
Our study indicates that the selective targeting by PEGliposomes of the inflamed synovial lining can strongly enhance the beneficial effects of GC in arthritis. The observed affinity of PEG-liposomes for macrophages may lead to high concentrations in inflammatory cells at arthritic joints combined with less exposure of healthy non-target tissues to GC.

\section{Authors' affiliations}

J M Metselaar, G Storm, Department of Pharmaceutics, Utrecht Institute of Pharmaceutical Sciences, The Netherlands

W B van den Berg, A E M Holthuysen, P L E M van Lent, Department of Rheumatology, University Hospital Nijmegen, Nijmegen, The Netherlands

M H M Wauben, Department of Infectious Diseases and Immunology, Division of Immunology, Faculty of Veterinary Medicine, Utrecht University, The Netherlands

J M Metselaar and G Storm are members of UNYPHAR, a network collaboration between the Universities of Groningen, Leiden, Utrecht and the pharmaceutical company Yamanouchi.

\section{REFERENCES}

1 Harris ED. Rheumatoid arthritis: pathophysiology and implications for therapy. N Engl J Med 1990;322:1277-89.

2 Bodman KB, Roitt IM. The pathophysiology of rheumatoid arthritis. Fund Am Clin Immunl 1994;2:73-81.

3 Van den Berg WB, Van Lent PLEM. The role of macrophages in chronic arthritis. Immunobiology 1996;195:614-23.

4 Van Lent PLEM, Holthuysen AEM, Van den Bersselaar L, Van Rooijen N, Van de Putte LBA, Van den Berg WB. Role of macrophage-like synovial lining cells in localization and expression of experimental arthritis. Scand J Rheumatol 1995:24(suppl 101):83-9.

5 Cutolo M, Sulli A, Barone A, Seriolo B, Accardo S. Macrophages, synovial tissue and rheumatoid arthritis. Clinical Exp Rheumatol 1993;11:331-9.

6 Kinne RW, Brauer R, Stuhlmuller B, Palombo-Kinne E, Burmester GR. Macrophages in rheumatoid arthritis. Arthritis Res 2000;2:189-202.

7 Van Rooijen N, Sanders A. Liposome mediated depletion of macrophages: mechanism of action, preparation of liposomes and applications. J Immunol Methods 1994;174:83-93.

8 Oussoren C, Storm G. Liposomes to target the lymphatics by subcutaneous administration. Adv Drug Deliv Rev 200;50:143-56. 
9 Ahsan F, Rivas IP, Khan MA, Torres Suarez Al. Targeting to macrophages: role of physicochemical properties of particulate carriers-liposomes and microspheres-on the phagocytosis by macrophages. J Control Release 2002;79:29-40.

10 Van Lent PL, Holthuysen AE, Van Rooijen N, Van De Putte LB, Van Den Berg WB. Local removal of phagocytic synovial lining cells by clodronateliposomes decreases cartilage destruction during collagen type II arthritis. Ann Rheum Dis 1998;57:408-13.

11 Barrera P, Blom A, van Lent PL, van Bloois L, Beijnen JH, van Rooijen $\mathrm{N}$, et al. Synovial macrophage depletion with clodronate-containing liposomes in rheumatoid arthritis. Arthritis Rheum 2000;43:1951-9.

12 Camilleri JP, Williams AS, Amos N, Douglas-Jones AG, Williams BD. Liposomal clodronate depletes synovial macrophages, suppresses inflammation and prevents radiological progression in adjuvant arthritis. Arthritis Rheum 1995;38(suppl 9):1319.

13 Richards PJ, Williams AS, Goodfellow RM, Williams BD. Liposomal clodronate eliminates synovial macrophages, reduces inflammation and ameliorates joint destruction in antigen-induced arthritis. Rheumatology (Oxford) 1999;38:818-25.

14 Richards PJ, Williams BD, Williams AS. Suppression of chronic streptococcal cell wall-induced arthritis in Lewis rats by liposomal clodronate. Rheumatology (Oxford) 2001;40:978-87.

15 Camilleri JP, Williams AS, Amos N, Douglas-Jones AG, Love WG, Williams BD. The effect of free and liposome-encapsulated clodronate on the hepatic mononuclear phagocyte system in the rat. Clin Exp Immunol 1995;99:269-75.

16 Joyce DA, Gimblett G, Steer JH. Targets of glucocorticoid action on TNFalpha release by macrophages. Inflamm Res 2001;50:337-40.

17 Amano Y, Lee SW, Allison AC. Inhibition by glucocorticoids of the formation of interleukin-1 alpha, interleukin-1 beta, and interleukin-6: mediation by decreased mRNA stability. Mol Pharmacol 1993;43:176-82.

18 Wong PK, Cuello C, Bertouch JV, Roberts-Thomson PJ, Ahern MJ, Smith MD, et al. Effects of pulse methylprednisolone on macrophage chemotactic protein1 and macrophage inflammatory protein-lalpha in rheumatoid synovium. $J$ Rheumatol $2001 ; 28: 2634-6$.

19 Cutolo M, Foppiani L, Prete C, Ballarino P, Sulli A, Villaggio B, et al. Hypothalamic-pituitary-adrenocortical axis function in premenopausal women with rheumatoid arthritis not treated with glucocorticoids. J Rheumatol 1999;26:247-50.

20 ACR. Guidelines for the management of rheumatoid arthritis. American College of Rheumatology Ad Hoc Committee on Clinical Guidelines. Arthritis Rheum 1996;39:713-22.

21 Saag KG. Glucocorticoid use in rheumatoid arthritis. Curr Rheumatol Rep 2002;4:218-25.
22 Snell ES. The pharmacological properties of corticosteroids in relation to clinical efficacy. Br J Dermatol 1976;94(suppl 12):15-23.

23 Woodle MC, Lasic DD. Sterically stabilized liposomes. Biochim Biophys Acta 1992;1113:171-99.

24 Oku N, Namba Y. Long-circulating liposomes. Crit Rev Ther Drug Carrier Syst 1994;11:231-70.

25 Woodle MC, Newman MS, Cohen JA. Sterically stabilized liposomes: physical and biological properties. J Drug Target 1994;2:397-403.

26 Laverman P, Boerman OC, Oyen WJ, Dams ET, Storm G, Corstens FH. Liposomes for scintigraphic detection of infection and inflammation. Adv Drug Deliv Rev 1999;37:225-35.

27 Metselaar JM, Wauben MH, Wagenaar-Hilbers JP, Boerman OC, Storm G. Complete remission of experimental arthritis by joint targeting of glucocorticoids with long-circulating liposomes. Arthritis Rheum 2003:48:2059-66.

28 Amselem S, Gabizon A, Barenholz Y. A large-scale method for the preparation of sterile and non-pyrogenic liposomal formulations of defined size distributions for clinical use. In: Gregoriadis G, ed. Liposome technology. Boca Raton, FL: CRC Press, 1993:501-25.

29 Rouser G, Fkeischer S, Yamamoto A. Two-dimensional thin layer chromatographic separation of polar lipids and determination of phospholipids by phosphorus analysis of spots. Lipids 1970;5:494-6.

30 Huang SK, Hong K, Lee KD, Papahadjopoulos D, Friend DS. Light microscopic localization of silver-enhanced liposome-entrapped colloidal gold in mouse tissues. Biochim Biophys Acta 1991;1069:117-21.

31 Courtenay JS, Dallman MJ, Dayan AD, Martin A, Mosedale B. Immunisation against heterologous type II collagen induces arthritis in mice. Nature 1980;283:666-8.

32 Weusten BLAM, Jacobs JWG, Bijlsma JWJ. Corticosteroid pulse therapy in active rheumatoid arthritis. Semin Arthritis Rheum 1993;23:183-92.

33 Roujeau JC. Pulse glucocorticoid therapy. The 'big shot' revisited. Arch Dermatol 1996;132:1499-502.

34 Liebling MR, Leib E, McLaughlin K, Blocka K, Furst DE, Nyman K, et al. Pulse methylprednisolone in rheumatoid arthritis: a double-blind cross-over trial. Ann Intern Med $1981 ; 94: 21-6$.

35 Hansen TM, Dickmeiss E, Jans $H$, Ingemann Hansen T, Ingeman-Nielsen $M$, Lorenzen I. Combination of methylprednisolone pulse therapy and remission inducing drugs in rheumatoid arthritis. Ann Rheum Dis 1987;46:290-5.

36 Schiffelers RM, Storm G, Bakker-Woudenberg IA. Host factors influencing the preferential localization of sterically stabilized liposomes in Klebsiella pneumoniae-infected rat lung tissue. Pharm Res 2001;18:780-7.

37 Laverman P, Dams ET, Storm G, Hafmans TG, Croes HJ, Oyen WJ, et al. Microscopic localization of PEG-liposomes in a rat model of focal infection. $\mathrm{J}$ Control Release 2001;75:347-55. 